\section{Blood flow in venules: A mathematical model including valves inertia}

\author{
Matteo Cardini, ${ }^{1}$ Angiolo Farina, ${ }^{1}$ \\ Antonio Fasano, ${ }^{1-3}$ Alberto Caggiati ${ }^{4}$ \\ ${ }^{1}$ Department of Mathematics and \\ Informatics, University of Firenze; \\ ${ }^{2}$ Scientific Manager and R\&D Director, \\ FIAB, Firenze; ${ }^{3}$ Associated to IASI- \\ CNR, Roma; ${ }^{4}$ Department of Anatomy, \\ La Sapienza University, Roma, Italy
}

\begin{abstract}
It is well known that venules equipped with valves play a critical role in regulating blood flow. Essentially they are peristaltic pumps that increase the efficiency of venous blood return to the heart, thanks to the presence of valves preventing backflow. Inspired by two recent papers, we have modeled the venule as a vessel with valves placed at its ends and walls animated by radial oscillations that are independent of heart pulsation and respiratory rhythm. Differently from the previous papers, the present model takes into account the valves inertia allowing, for progressive closing/opening stages. The numerical simulations produce a pressure pulse and a velocity profile which agree almost perfectly with the experimental data of Dongaonkar et al., eliminating the discrepancies found in Farina et al., arising from the hypothesis that valves act instantaneously.
\end{abstract}

\section{Introduction}

The phenomenon of vasomotion consists in periodic oscillations of blood vessels walls and it was studied for the first time by Thomas Wharton Jones ${ }^{1}$ in 1852 . He observed spontaneous oscillations of the venules in the batwing membrane and rightly concluded that its effect was to enhance blood flow. Also Intaglietta ${ }^{2}$ pointed out the effects of vasomotion on blood flow in small vessels, distinguishing between the arterioles and the venules. Gratton et al. ${ }^{3}$ made experiments on pregnant rats and they concluded that vasomotion has many potential functions, including modulation of vascular resistance. In particular, the authors claimed that the arterioles hydraulic resistance increases as the amplitude of vasomotion increases and this result appears consistent with experimental observations. The whole matter of blood dynamics in the presence of vasomotion has been recently reconsidered in Farina et al. ${ }^{4}$ and Fasano et al., ${ }^{5}$ where the authors made a clear distinction between the flow in the venules and in arterioles. In a recent paper Dongaonkar et $a l .{ }^{6}$ experimenting on batwings focused on the presence of valves in venules. Batwings are characterized by a very thin membrane allowing in vivo, non-invasive, nondestructive measurements with simple light microscopy without the use of anesthesia or surgery.

Looking at Fig. 3 of Dongaonkar et al. ${ }^{6}$ we immediately realize the oscillating behavior of the venule walls and the absence of retrograde flow. Moreover, such oscillations are unrelated to heart pulsations since their period is too long (almost $7 \mathrm{~s}$ ) and they act as a peristaltic pump, with valves preventing the back-flow. Thus, the peristaltic action results in pressure pulses greatly enhancing the effect of the heart generated hydraulic pressure gradient. As a consequence the vessel resistance is considerably reduced, as shown in Farina et al. ${ }^{4}$ Indeed, by preventing reflux, microvalves turn the periodic oscillations of venules walls into a pumping action. In the contraction phase the rear valve closes, while expansion causes the closure of the front valve, thus making flow unidirectional. In valveless vessels (like arterioles) vasomotion scarcely influences the net blood discharge (and in a negative way in a purely Newtonian framework ${ }^{5}$ ). It is frequently believed that valves are absent in human body veins smaller than two millimeters in diameter and so investigations on the pathophysiology of chronic venous diseases normally consider and evaluate only the vascular competence of large veins. On the contrary, reviewing literature (see, Caggiati et $a l .^{7}$ and the recent book by Fasano and Sequiera $^{8}$ ) it turns out that the discovery of microscopic venous valves dates almost a hundred years ago.

The mechanisms triggering vasomotion are discussed in a number of papers. We refer the reader to the reviews by other authors $^{9-11}$ and to the numerous references therein. However the focus of this paper is not on the mechanisms that cause and control vasomotion, but rather on the consequences that vasomotion has on the blood flow in venules equipped with compliant valves.

A mathematical model for the venular flow recorded by Dongaonkar et al., ${ }^{6}$ has been formulated and studied in Farina et al. ${ }^{4}$ and Fasano et al., ${ }^{5}$. Such a model describes peristaltic effect arising from the combination of the walls oscillations and of the valves action fitting the experimental data Dongaonkar et al. ${ }^{6}$ reasonably well. The discrepancy between the predicted and the
Correspondence: Angiolo Farina, Department of Mathematics and Informatics, University of Firenze, viale Morgagni 67/A, 50134 Firenze, Italy. Tel./Fax: +39.055.2751436.

E-mail: angiolo.farina@unifi.it

Key words: Vasomotion; blood flow in venules; mathematical modeling.

Received for publication: 23 November 2018. Revision received: 27 February 2019.

Accepted for publication: 28 February 2019.

This work is licensed under a Creative Commons Attribution 4.0 License (by-nc 4.0).

(Copyright: the Author(s), 2019

Licensee PAGEPress, Italy

Veins and Lymphatics 2019; 8:7946

doi:10.4081/vl.2019.7946

experimental vasomotion-related pressure peaks consists in a retardation of the pressure-ascending phase and in an anticipation of the descending branch. We believe that the underlying cause is that the time taken by the valves to open or close was simply ignored. Indeed, in Farina et al. ${ }^{4}$ and Fasano et al. ${ }^{5}$ valves were modeled as massless bodies which open/close as the pressure in the venule becomes larger/smaller than the one outside. In other words, valves inertia was neglected.

The purpose of this study is to propose a simple model, which accounts effectively for valves inertia. The valves, indeed, are driven by the flow in a complicated way and their response can never be instantaneous, due to their mass. We believe that the model here proposed, though tentative, can be useful because explains qualitatively and quantitatively how vasomotion affects blood flow in venules, giving an excellent agreement with the experimental data. Further, the model is also able to reproduce the effects of some pathologies like the partial incontinence of the valves.

The fitting procedure has been developed with the available data by Dongaonkar et al., ${ }^{6}$ and its purpose is to show how the model is able to reproduce the experiments. The pressure data that we consider in this paper refer to the venular flow in bat wing. It could be interesting to compare our results with human venules, especially with pathological cases. However, to the best of our knowledge, these data are not available. Nevertheless, inserting the parameters concerning human venules vasomotion (vessels size, oscillation period and amplitude) one could make some predictions about the effect of this phenomenon. 


\section{Materials and Methods}

We consider a cylindric vessel whose length is $L^{*}$ and whose radius oscillates with period $T^{*}$ (typically $T^{*} \approx 6-7 \mathrm{~s}$ for bats), namely $R^{*}=R_{0}^{*} R\left(t^{*}\right)$, with $R_{0}^{*}$ maximum radius and $R\left(t^{*}\right)$ dimensionless periodic function such that $(1-2 \delta) \leq R\left(t^{*}\right) \leq 1$, with $\delta<0.5$ [Note: all symbols with $*$ denote dimensional quantities.]. We remind that vasomotion period is not related to heart rhythm, as pointed out by Intaglietta in his 1990 paper. $^{2}$

The ratio $\varepsilon=\frac{R_{0}^{*}}{L^{*}}$ and the Reynolds number $R e$ are small (typically $\varepsilon=2.5 \times 10^{-3}$ and $R e \leq 10^{-4}$ ) and this allows writing the flow equations in a greatly simplified form. ${ }^{4}$ Actually, the limitations of the model are essentially linked to these two hypothesis: creeping flow $(\operatorname{Re}<<1)$ and lubrication regime $(\varepsilon<<1)$.

We remark that we consider synchronous oscillations, i.e. $R^{*}=R^{*}\left(t^{*}\right)$, and not a peristaltic progressive wave, for the following reasons. First in Dongaonkar et al. ${ }^{6}$ there is no information on the phase velocity (or, equivalently, on the wavelength $\lambda^{*}$ ). The authors report only the oscillations frequency and amplitude. Next the effects of the peristaltic wavelength $\lambda^{*}$ on the flow have been analyzed by Farina et $a l .{ }^{4}$ and Fusi et $a l .{ }^{12}$ In these papers three cases have been considered: i) $\lambda^{*}$ much smaller than the vessel length $L^{*}$; ii) $\lambda^{*}$ of the same order of $L^{*}$; iii) $\lambda^{*}$ much larger than $L^{*}$. Case i) is extremely disadvantageous for the flow, resulting in a sharp increase of the vessel hydraulic resistance. In case ii) the fit with the experimental data ${ }^{6}$ is very poor. So, the only interesting case turns out to be case iii), which basically corresponds to synchronous oscillations.

We also suppose that the inlet-outlet pressure difference $\Delta p^{*}$ is known and we introduce $\Delta p=\frac{\Delta p^{*}}{p_{r e f}^{*}}, p^{*}{ }_{\text {ref }}$ being the reference pressure obtained from Poiseuille formula (exploiting the data of Dongaonkar et al., ${ }^{6}$ $p^{*}{ }_{\text {ref }}=0.37 \mathrm{cmH}_{2} 0$ ). We indeed remark that the flow is driven by the hydraulic pressure gradient due to heart to which the peristaltic pressure is superimposed. The oscillation of the vessel walls provides an active venular pumping thanks to the presence of compliant valves (a characteristic of reciprocating pumps), which prevent retrograde flow.

For simplicity we consider the blood to be a Newtonian fluid whose viscosity is $\mu^{*}$ $=3.5 \mathrm{mPa} \cdot s$. In Cardini ${ }^{13}$ a power-law model is consider but numerical simulations have pointed out such a non-Newtonian behavior does not improve the fit with the experimental data of Dongaonkar et al. ${ }^{6} \mathrm{We}$ therefore conclude that the simple Newtonian model is satisfactory for our scope.

The dimensionless space and time variables are:

$$
x=\frac{x^{*}}{L^{*}}, \quad r=\frac{r^{*}}{R_{0}^{*}}, \quad t=\frac{t^{*}}{T^{*}}
$$

where $x^{*}$ and $r^{*}$ are the longitudinal and the radial coordinate, respectively.

We also introduce the dimensionless pressure:

$$
\begin{aligned}
& p=\frac{p^{*}\left(x^{*}, t^{*}\right)-p^{*}\left(L^{*}, t^{*}\right)}{p_{\text {ref }}^{*}}, \\
& \text { where } \frac{p_{\text {ref }}^{*}}{L^{*}}=\frac{v_{i, r e f}^{*} \mu^{*}}{R_{0}^{* 2}}, \text { so that }\left.p\right|_{\text {inlet }}= \\
& \Delta p=\frac{p^{*}\left(0, t^{*}\right)-p^{*}\left(L^{*}, t^{*}\right)}{p_{\text {ref }}^{*}}, \text { and }\left.p\right|_{\text {outlet }}=0 .
\end{aligned}
$$

The analysis developed in Farina et al. ${ }^{4}$ provides for the differential equation:

$$
\frac{\partial^{2} p(x, t)}{\partial x^{2}}=\frac{16}{\delta R^{3}(t)} \frac{d R(t)}{d t}
$$

Whose solution is:

$$
p(x, t)=\frac{8}{\delta R^{3}(t)} \frac{d R(t)}{d t} x^{2}+A(t) x+B(t)
$$

where $A(t)$ and $B(t)$ have to be determinated using the conditions imposed at the vessel ends. From (3) we can compute the dimensionless discharge $Q$, getting:

$$
\begin{aligned}
& Q(x, t)=-\frac{\pi}{8} \frac{\partial p(x, t)}{\partial x} R^{4}(t)= \\
& -\frac{\pi}{8}\left(\frac{16}{\delta R^{3}(t)} \frac{d R(t)}{d t} x+A(t)\right) R^{4}(t)
\end{aligned}
$$

and the centerline blood longitudinal velocity:

$$
\begin{aligned}
& \left.v_{1}\right|_{r=0}=-\frac{1}{4} \frac{\partial p}{\partial x} R^{2}(t)= \\
& -\frac{1}{4}\left(\frac{16}{\delta R^{3}(t)} \frac{d R(t)}{d t} x+A(t)\right) R^{2}(t) .
\end{aligned}
$$

The valves dynamics excluding inertia (model adopted by in Farina et $a l^{4}{ }^{4}$ and Fasano et $\left.a l,{ }^{5}\right)$ is described by the following two steps:

i) the inlet valve $(x=0)$ closes when the pressure caused by the vessel contraction exceeds the imposed one, i.e. $\Delta p$. This fact prevents the back-flow, forcing the discharge $Q$ to vanish when $\left.p\right|_{x=0}>\Delta p$.

ii) the outlet valve $(x=1)$ closes when pressure falls below the outlet one (which we have set equal to 0 ).

These conditions allow to find $A(t)$ and $B(t)$ and eventually, exploiting (3) and (4), to get an explicit formula for pressure and discharge. In particular, conditions 1 and 2 are graphically represented by the step functions (solid lines in Figure 1 parallel to axes).

However, in this way the valves are modeled as mass-less devices which open/close instantaneously as the pressure in the vessel becomes larger/smaller than the one outside. Such an approach, though providing a significant agreement with the experiments, is now improved by somehow taking into account the valves inertia, which induces a delay in their action. An effective way of representing this delay is to smooth the original boundary conditions 1 and 2 , replacing them with:

$\left.p\right|_{x=0}=\Delta p \frac{\left(\left.\frac{\partial p}{\partial x}\right|_{x=0}\right)-n}{\left.\frac{\partial p}{\partial x}\right|_{x=0}}$,

and

$$
\left.p\right|_{x=1}=\frac{m}{\left.\frac{\partial p}{\partial x}\right|_{x=1}}
$$

$n, m$ being two parameters characterizing the opening/closing speed. Thus, once
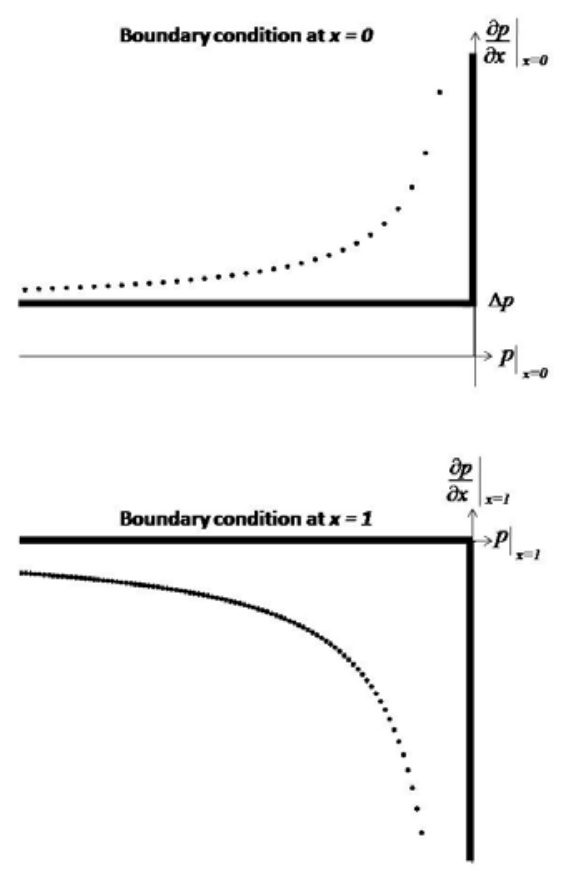

Figure 1. Boundary conditions at $x=0$ and $x=1$ (pressure gradient vs pressure). The solid lines represent the instantaneous open/close conditions, while the dotted lines are their smoothed version. 
given the radius oscillation profile $R(t)$, we have to couple (6) and (7) with (3), in order to numerically determine $A(t)$ and $B(t)$. Then formulas (4) and (5) will provide the explicit expression for the respective quantities.

We remark that an accurate description of valves dynamics would require quantitative information about their mechanical properties, such as their mass, shape, stiffness in addition to their interaction with flowing blood. As stated earlier, such extremely complex dynamics has been bypassed in previous papers, ${ }^{4,5}$ by assuming that valves have a simple on/off behavior (i.e. they open and close immediately when requested). Since the actual study of the valves dynamics is far beyond our possibility of investigation, we came to the compromise of mimicking the implied delay by introducing some progressive way in the opening and closing, which in the model amounts in replacing the step functions (the solid lines in Figure 1) with smooth functions (the dotted lines in Figure 1) characterized by the parameters $m$ and $n$. Though they do not have a precise physical meaning, they allow introducing in the model the effects of valves reduced efficiency due to their inertia and, in extreme cases, to pathological reasons.

\section{Results and Discussion}

Omitting mathematical technicalities, we illustrate the main results (the readers are referred to Cardini ${ }^{13}$ where mathematical details are illustrated). We select:

$R(t)=2.37 t^{3}\left(1-t^{3}\right)^{3}+0.75$, repeated periodically with period $T=1$,

and take $R_{0}^{*}=70 \mu \mathrm{m}, T=6 \mathrm{sec}$, fitting very well the oscillation of $R^{*}$ shown in Fig. 3 of Dongaonkar et al. ${ }^{6}$ Next, we exploit equations (6) and (7) to get the parameters $A(t)$ and $B(t)$ appearing in (3) and (5).

The dashed line in Figure 2 is the pressure pulse produced by the present model (with $n=100$ and $m=50$ ), the continuous line is the one produced by the previous illustrated $\operatorname{model}^{5}$ and the asterisks are the experimental data. ${ }^{6}$

Clearly, the model presented here matches the experimental data ${ }^{6}$ better than which disregarded valves inertia, confirming at the same time that the two-valve model is reliable and that valves inertia have a substantial influence.

Moreover, the model predicts that, during a certain time, both valves are open. This occurs when the lumen diameter is close to its maximum or minimum. In this stage, that we can call inert phase, both valves are open and the blood flow is simply driven by the pressure difference generated by the heart.

We observe further that the model can be used for making predictions about pathological cases as shown in Figure 3. For instance, in the case of varicose the vasomotion ceases or is reduced owing to the wall relaxation, as in Figure 3A where the oscillation amplitude is reduced by $30 \%$. The reduction of vasomotion entails in turn an appreciable reduction of pumping action, Figure $3 \mathrm{~B}$, associated to the spontaneous oscillation of the venous walls.

Valves incontinence can be simulated by a partial opening/closure, which can be effectively represented by further slowing the valves action. In Figure 4 we have plotted the inlet discharge corresponding to efficient valves (continuous line) and incontinent valves (dashed line). In this case back flow (namely negative discharge) occurs during the compression stage and the net discharge practically vanishes. The inlet valve does not sustain the pressure generated by the contraction of the vessel wall and this generates the backflow (which is absent in the case of healthy valves).

\section{Conclusions}

As shown in Chen et al., ${ }^{14}$ the role of the valves is not only to prevent reflux but also to increase the out flow. And also the insufficiency of the microvalves determines both

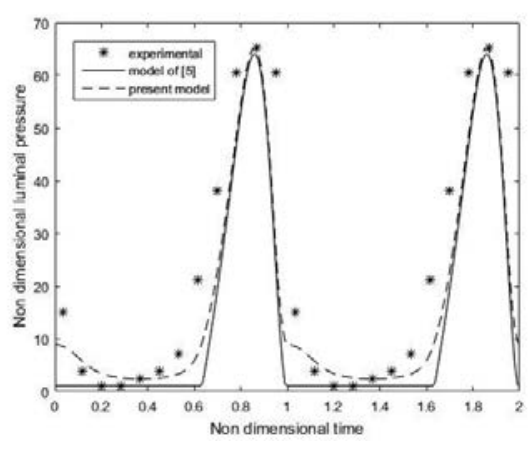

Figure 2. Vasomotion-induced pressure pulse. Comparison between the experimental data of Dongaonkar et al.6 (asterisks), the model illustrated in Fasano et al. ${ }^{5}$ (solid line), and the model developed in this paper (dashed line) with $n=100$ and $m=50$. In order to better appreciate the differences two periods are shown. micro reflux (see, for instance, a recent paper by Govind et al. ${ }^{15}$ ), and reduction of the venular outflow. The model agrees qualitatively with these results. For instance, the inlet discharge of valves whose opening/closure is reduced by $70 \%$, decreases by about $95 \%$ with respect to the healthy case.

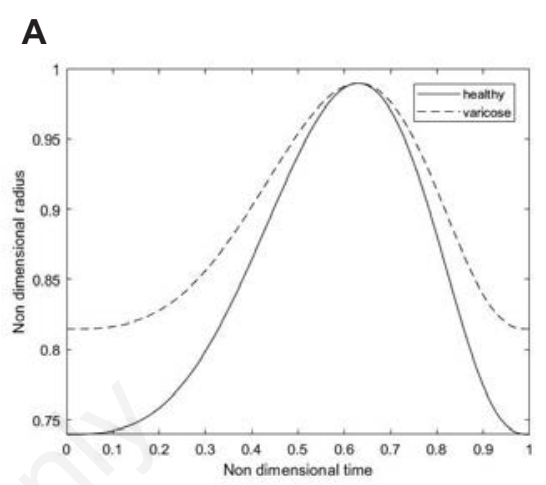

B

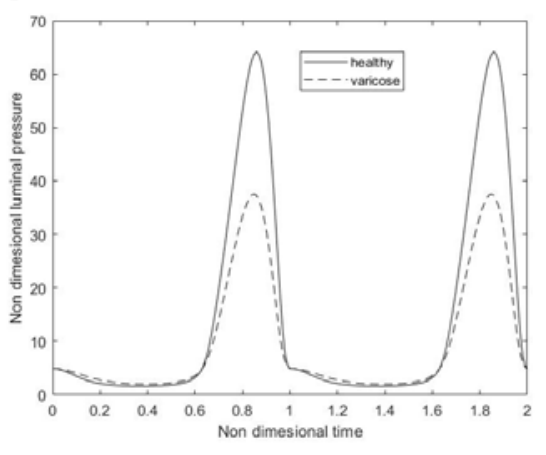

Figure 3. Comparison between healthy and varicose vasomotion. A) Radius oscillation in healthy condition (continuous line) and in the presence of a varicose pathology (dashed line). B) Pressure pulse in healthy condition (continuous line) and in the presence of a varicose pathology (dashed line).

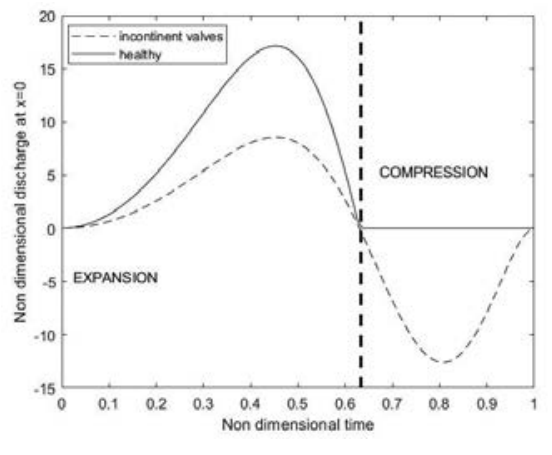

Figure 4. Inlet discharge in case of efficient valves (continuous line) and incontinent valves (dashed line) whose opening/closure is reduced by $70 \%$. The net discharge is reduced by about $95 \%$ compared to the healthy valve case. 
Possible future developments could concern a more accurate investigation of the energetic benefits that vasomotion brings to the circulatory system. For example, which additional effort is required to the heart in case of pathology affecting valves? This could give important qualitative information on the cardiac effects of venous diseases.

\section{References}

1. Jones TW. Discovery that the veins of the bat's wing are endowed with rhythmical contractility and that onward flow of blood is accelerated by each contraction. Philos Trans Royal Soc B: Biol Sci 1852;142:131-6.

2. Intaglietta M. Vasomotion and flowmotion: physiological mechanisms and clinical evidence. Vasc Med Rev 1990;1:1101-12.

3. Gratton RJ, Gandley RE, McCarthy JF, et al. Contribution of vasomotion to vascular resistance: a comparison of arteries from virgin and pregnant rats. J Appl Physiol 1998;85:2255-60.

4. Farina A, Fusi L, Fasano A, et al. Modeling peristaltic flow in vessels equipped with valves: implications for vasomotion in bat wing venules. Int $\mathrm{J}$ Eng Sci 2016;107:1-12.

5. Fasano A, Farina A, Caggiati A. Modeling vasomotion. Rev Vasc Med 2016;8:1-4.

6. Dongaonkar RM, Quick CM, Vo JC, et al. Blood flow augmentation by intrinsic venular contraction in vivo. Am $\mathrm{J}$ Physiol Regul Integr Comparat Physiol 2012;302:R1436-42.

7. Caggiati A, Phillips M, Lametschwandtner A, Allegra C. Valves in small veins and venules. Eur J Vasc Endovasc Surg 2006;32:447-52.

8. Fasano A, Sequeira A. Hemomath: The Mathematics of Blood. Berlin: Springer; 2017.

9. Reho JJ, Zheng X, Fisher SA. Smooth muscle contractile diversity in the control of regional circulations. Am J Physiol Heart Circ Physiol 2014;306:H163-72.
10. Haddock RE, Hill CE. Rhythmicity in arterial smooth muscle. J Physiol 2005; 56:645-56.

11. Aalkjaer C, Nilsson H. Vasomotion: cellular background for the oscillator and for the synchronization of smooth muscle cells. Br J Pharmacol 2005;144: 605-16.

12. Fusi L, Farina A, Fasano A. Short and long wave peristaltic flow: modeling and mathematical analysis. Int J Appl Mechanics 2015;7:1550014.

13. Cardini M. Blood flow in venules: a mathematical model. Master's Degree Thesis. Università degli Studi di Firenze; 2017. Available from: https:// sol.unifi.it/tesi/consultazione.

Chen HY, Diaz JA, Lurie F, et al. Hemodynamics of venous valve pairing and implications on helical flow. J Vasc Surg Venous Lymphat Disord 2018;6: 517-22.

15. Govind D, Thomas KN, Hill BG, van Rij AM. Microvenous reflux in the skin of limbs with superficial venous incompetence. Ultrasound Med Biol 2018;44: 756-61. 